\title{
Cell death research, on an island girt by sea
}

\author{
ME Christensen ${ }^{1}$, WW-L Wong ${ }^{2,3}$, M Waibel ${ }^{4}$, RW Johnstone ${ }^{4,5,6}$ and NJ Waterhouse ${ }^{\star, 1,7}$ \\ Cell Death and Differentiation (2012) 19, 1090-1091; doi:10.1038/cdd.2012.32; published online 16 March 2012
}

1st Australian Workshop, on cell Death: Death on the Reef in Lindemann Island, Australia, 21-25 August 2011

\begin{abstract}
As the number of Australians who attended the fifth European Workshop on Cell Death (EWCD) was sufficient to field a soccer team capable of taking on the rest of the World, it seemed fitting that we should host an Australian version. 'Death on the Reef', the First Australian WCD was therefore held in August 2011 on a secluded island location off the coast of Queensland to discuss the latest research in this field. The meeting attracted predominantly young scientists who presented short talks on their current research, and highlighted that not only does Australia, 'abound in nature's gifts', as proclaimed by the national anthem, but also abounds in high quality cell death research. Although it was not possible to cover all of the talks here because of editorial restrictions, the quality of these talks made a good argument for a biennial meeting to complement the EWCD.
\end{abstract}

\section{Drilling Down on Death Receptors}

Ligation of death receptors is an efficient way to kill cancer cells, but what makes a cancer cell sensitive to death receptor ligation? Where is the commitment to death? And do we know all of the players? The Borst group (The Netherlands) screened siRNA libraries to identify proteins that alter sensitivity of cells to TRAIL and found two novel determinants (SRP-72 and MARCH-8) involved in regulating TRAIL-R1 expression. Lynn Wong (WEHI) showed that knocking down inhibitor of apoptosis proteins (IAPs) with Smac mimetics killed cells in a TNF dependent manner that required both RIPK1 and RIPK3, and also triggered elevated levels of cytokines that are consistently observed in the clAP1/ cIAP2/XIAP triple knockout mice. Nufail Khan (La Trobe) showed that these Smac mimetics also synergised with IFN $\gamma$ to trigger cell death in a RIPK1-dependent manner, suggesting that combining TNF or IFN $\gamma$ with Smac mimetics could be used to treat patients with cancers that are resistant to either treatment alone. In contrast, Donia Moujalled (La Trobe) showed that RIPK3 sensitizes cells to TNF-induced cell death by a mechanism that did not involve RIPK1, suggesting that although they use similar molecules, the signaling pathways initiated by TNF and IFN $\gamma$ are likely to be intrinsically different. It is therefore clear that there is still much to learn about pathways activated by ligation of death receptors and when they trigger apoptosis or necroptosis.

\section{Bidding on Your Favorite Bcl-2 Family Member}

Ligation of death receptors is also known to activate the mitochondrial pathway to apoptosis, and Joanne O'Donnell (WEHI) used time-lapse microscopy to investigate how Bcl-2 family members that regulate Fas-induced death in neutrophils. Her studies showed that overexpression of Bcl-2 or Mcl-1 could delay death by several hours but were not required for normal survival, suggesting that $\mathrm{Bcl}-2$ and $\mathrm{Mcl}-1$ may contribute to disease by increasing the longevity of these cells. The pressures generated by pro-apoptotic $\mathrm{Bcl}-2$ family members are also essential for cell death in other models, and one question is whether the strength of signal will impact on cell fate, disease progression and treatment strategies. Delphine Merino (WEHI) investigated the strength of the pro-apoptotic signal in vivo generated by the $\mathrm{BH} 3$ domains of Puma, Noxa and Bad swapped into the $\mathrm{Bim}-\mathrm{BH} 3$ genetic locus. These studies showed that the $\mathrm{BH} 3$ domain of Bim offered the strongest signal followed by Puma, Noxa and finally Bad in a model of immune cell homeostasis. The BH3 domains of Puma or Bim were superior to the $\mathrm{BH} 3$ domains of Bad or Noxa in a tumorigenic model; and all of the Bim mutants, except Bim with the $\mathrm{BH} 3$ domain of Bad, were equally strong in killing Bcl-2 overexpressing cells in response to ABT-737. The strength of signal of $\mathrm{BH} 3$ domains in determining cell fate, disease progression and response to therapy is therefore likely to be context and cell type specific.

\footnotetext{
${ }^{1}$ Apoptosis and Cytotoxicity Laboratory, Mater Medical Research Institute, South Brisbane, Queensland, Australia; ${ }^{2}$ Cell Signaling and Cell Death, The Walter and Eliza Hall Institute of Medical Research, Parkville, Victoria, Australia; ${ }^{3}$ Institute of Experimental Immunology, University of Zürich, Zürich, Switzerland; ${ }^{4}$ Gene Regulation Laboratory, Cancer Therapeutics Program, Peter MacCallum Cancer Centre, Melbourne, Victoria, Australia; ${ }^{5}$ Department of Microbiology and Immunology, University of Melbourne, Melbourne, Victoria, Australia; ${ }^{6}$ Victorian Comprehensive Cancer Centre, Parkville, Victoria, Australia and ${ }^{7}$ Department of Medicine, University of Queensland, St Lucia, Queensland, Australia

${ }^{*}$ Corresponding author: NJ Waterhouse, Apoptosis and Cytotoxicity Laboratory, Mater Medical Research Institute, Aubigny Place, Raymond Terrace, South Brisbane, Queensland 4101, Australia. Tel: + 6173163 3888; Fax: + 6173163 2550; E-mail: nwaterhouse@mmri.mater.org.au
} 


\section{Targeting Bcl-2 Family Members in Cancer}

Various studies showed that it may not be sufficient to only look at the effect of one Bcl-2 family member when trying to predict therapeutic responses. Michaela Waibel (Peter Mac) showed that expression of $\mathrm{Bcl}-2, \mathrm{Bcl}-\mathrm{xL}$ and $\mathrm{Bim}$ is controlled by the hyperactive JAK2 tyrosine kinase in JAK2-driven acute lymphoblastic leukemias. The combined approach to inhibit Bcl-2/Bcl-xL using ABT-737, and increasing Bim expression by inhibiting JAK2 activity induced longterm remissions in mice bearing JAK2-driven T-cell leukemia, revealing a new approach for treating hematological cancers driven by mutated JAK2. Stefan Glaser (WEHI) also showed that removal of $\mathrm{Mcl}-1$, but not $\mathrm{Bcl}-\mathrm{xL}$, could cause cell death in mice afflicted with acute myeloid leukemia (AML), whereas forced expression of $\mathrm{Mcl}-1$ has a critical role in survival of $\mathrm{AML}$ cells. Bim peptides, which bind to all of the anti-apoptotic Bcl-2 family members, may therefore prove effective for treatment for these cancers, or where increased expression of Mcl-1 has contributed to relapse. Melinda Christensen (MMRI) investigated the potential for $\mathrm{Mcl}-1$ to cause relapse in cancer patients treated with immunotherapy. Her research showed that although $\mathrm{Bcl}-2$ could block the mitochondrial pathway during granule-induced death, Mcl-1 did not. This suggests that immunotherapy is still a viable option for patients with relapse following chemotherapy because of increased levels of $\mathrm{Mcl}-1$.

\section{Immunity, The Big $C$ and Beyond}

Helen Beere (USA) heated things up using knockout models to show a new role for heat shock protein 70 (HSP-70) in tumor cell clearance by promoting anti-tumour immunity. A similar effect was also observed by Steven Matarollo (Peter Mac) who showed that specific subsets of cytotoxic T cells infiltrated the tumor microenvironment of MCA-induced tumors treated with doxorubicin. Understanding how HSP-70 and chemotherapy co-ordinate with cytotoxic cells to kill cancer cells may give us essential clues for harnessing the immune response to increase the efficacy of current therapies.

Cytotoxic cells are required for killing tumor cells by a mechanism that involves perforin and granzymes. Consistent with this, Jenny Chia (Peter Mac) showed that mutations that impair perforin activity may be the underlying cause of some late onset hematological malignancies. Dawn Cooper (Canada) showed that extracellular granzyme $\mathrm{K}$ can alter inflammatory cytokine and chemokine production, and may provide a mechanism by which granzymes modulate recruitment of immune cells. David Granville (Canada) also showed that granzyme B can cleave decorin, an important molecule in collagen organization, which leads to atherosclerosis. In this way, granzyme B may have a positive role in cancer cell

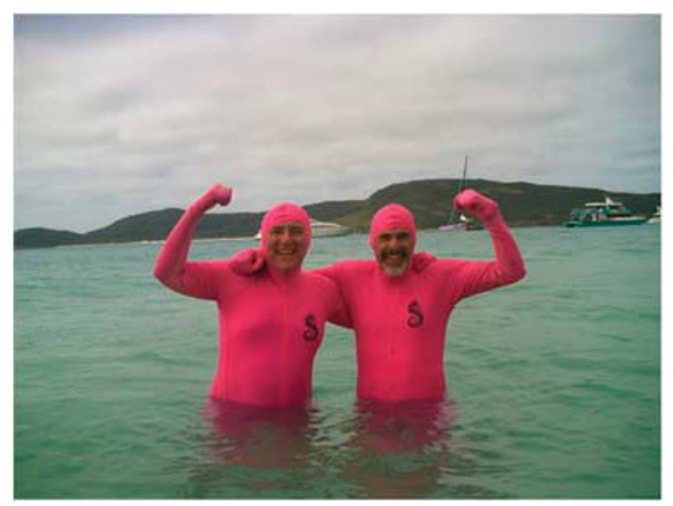

Figure 1 Doug Green and Gerry Melino visit the Great Barrier Reef dressed as teletubbies

death but may also have a more undesirable role that warrants further investigation.

Ueli Nachbur (WEHI) was also 'Lub-ing' up potential reviewers and 'Hoip-ing' to get papers in Cell Death and Differentiation by investigating the role of cell death also has a role in diseases other than cancer. Investigating the role of XIAP in FasL-induced hepatitis, Ueli showed that XIAP ubiquitinates RIPK2, allowing for recruitment of HOIL, HOIP and sharpin, a complex known as linear ubiquitin assembly complex (LUBAC). This results in full activation of NF- $\kappa B$ and MAP kinases, and mutations in the ring of XIAP found in XLP-2 patients results in impaired NF- $\kappa$ B and MAP kinase activation, reduced cytokine production and impairment of the immune response.

\section{A Nod to Climate Change in Australia}

'Death on the Reef' was a warming experience with delegates covering a diverse range of topics on most areas of cell death. As those who travelled to the conference will attest the floods that washed through Queensland earlier in the year did not dampen the spirits and enthusiasm of the nearly 100 delegates who visited this beautiful part of the world (Figure 1). Unfortunately Club Med Lindeman Island closed in January 2012. While we may never know whether that was our fault for drinking the bar dry or because of the recent weather patterns across Australia, we look forward to the next AWCD in 2013 in a similar exotic venue.

\section{Conflict of Interest}

The authors declare no conflict of interest.

Acknowledgements. The authors would like to acknowledge John Silke for his valuable comments on the manuscript. 